\title{
FLUCTUATION PHENOMENA AND STOCHASTIC PROCESSES
}

$\mathrm{T}$ HE theory of probability developed as a branch of pure mathematics. Its applications to physics have now become so widespread that there is scarcely a branch to which it does not contribute significantly. During the nineteenth century the establishment of the statistical nature of the second law of thermodynamics, the resolution of the irreversibility paradox, and the development of the powerful technique of statistical mechanics were all the result of applying statistical methods to an atomic population. In the twentieth century the wave-particle paradox was solved by rooting atomic physics in the theory of probability. At a less fundamental level, Brownian movement, diffusion and radio noise are physical phenomena the character of which is essentially stochastic ; and radio-wave propagation, sea waves, nuclear reactors and polymer physics are examples of fields in which stochastic problems have recently attracted considerable attention.

The apparatus of the mathematician has been accepted with gratitude by the physicist : generating functions and characteristic functions for manipulating probability distributions; generalized Fourier analysis introducing autocorrelation functions, and leading to the Wiener-Khintchine theorem for fluctuation phenomena stationary in time. In return the physicist has continually thrown up a variety of novel problems to challenge the ingenuity of the mathematician and maintain his interest.

The two-day conference of the Physical Society on "Fluctuation Phenomena and Stochastic Processes", held at Birkbeck College on March 19 and 20, attracted research workers in many different fields. Altogether, twenty-nine papers were presented; most of the participants were British, although contributions also came from the United States, Canada and Norway.

In his opening remarks of welcome, Prof. J. D. Bernal (Birkbeck College, London) pointed out that physics had learnt to deal adequately with the completely regular, and the completely random; it was the partially regular which still awaited treatment. The nature of the liquid state, and biophysical problems connected with the structure of large molecules, were important examples of this.

In the opening paper, Prof. M. S. Bartlett (University of Manchester) reviewed the various types of statistical fluctuations which occurred in physics, and attempted to classify them in order of relative size. In spite of the existence of occasional abnormal fluctuations, macroscopic averages in classical statistical mechanics had a 'stability' resulting from the large number of component systems involved. The measurement of time and space averages for phenomena such as turbulence and random surface waves, on the other hand, made use of the ergodic properties of some stationary processes. A fairly general 'weighted sum' type of process occurring in noise and Brownian motion theory was defined, together with the conditions for statistical 'stability'. Finally, there was the class of possibly exponentially increasing and unstable multiplicative (branching) processes such as nuclear cascades.

Dr. R. Furth (Birkbeck College) gave a comprehensive paper entitled "Fluctuations of Macroscopic
Parameters". Macroscopic parameters $\xi_{i}$ were operationally defined in finite regions $\Delta s$, of space and finite intervals $\Delta t$, of time of such magnitude that irregular fluctuations could be observed superimposed over the regular quasi-continuous functions $\xi_{i}(r, t)$. The theory of these fluctuations was mainly concerned with the determination of the second moments of the temporal fluctuations of the parameters $\xi_{i}$ in a fixed $\Delta s$, and of the spatial fluctuations in one and the same $\Delta t$. It was a characteristic feature of the theory that these moments could be expressed in terms of the macroscopic functions, $\xi_{i}(r, t)$, and that only some very general statistical properties of the random molecular processes responsible for the fluctuations needed to be known.

In the temporal problem the correlation functions of the type $c_{s}(\tau)=\left[\overline{\xi_{i}(\tau) \xi_{j}(t+\tau)}\right]_{s} /\left(\overline{\xi_{i} \xi_{j}}\right)_{s}$ could be calculated by making use of a generalized 'Langevin equation'; in the limiting case of $\tau=0$ the second moments $\left(\overline{\xi_{i} \xi_{j}}\right)_{s}$ might be obtained under conditions of statistical stationarity from statistical mechanics. This latter procedure could be applied to the problem of fluctuations of strain and stress in crystalline solids.

In the problem of spatial fluctuations the same method of statistical mechanics could be used for evaluating the spatial correlation products $\left[\overline{\xi_{i}(x) \xi_{j}(x+\zeta)}\right]_{t}$ of two parameters in two regions $(\Delta s)_{1}$ and $(\Delta s)_{2}$ in a homogeneous medium, separated by a distance $\zeta$ under conditions of 'quasi-stationarity', that is, when in spite of the finite speed of propagation of the interaction processes the values $\xi(x)$ and $\xi_{j}(x+\zeta)$ could be assumed to be simultaneous to a sufficient degree of approximation. This method could be used to obtain formulæ for the fluctuations of electric charge and potential in a discontinuous system of conductors; and had been applied by $\mathbf{E}$. Morris to the problem of fluctuations of surface charge density on the surface of a single continuous conductor, and the fluctuations of potential on and outside its surface.

Finally, the general problem of spatial fluctuations could be reduced to that of temporal fluctuations in such cases where the relevant Langevin equation had the character of a wave equation. This method was used by M. N. Moore for the solution of the problem of spatial fluctuations of strain and stress in erystalline solids.

Several papers dealt with the mathematical properties of stochastic functions, and with their application to the analysis of experimental data. Mr. D. G. Brennan (Massachusetts Institute of Technology, Lincoln Laboratory) in a paper entitled "A New Approach to Certain Types of Random Functions" developed $a b$ initio a theory of a class of stochastic processes which he hoped would have application to certain types of physical problem. Mr. M. B. Priestley (University of Manchester) considered the problem of detecting a signal containing several harmonic components in the presence of background noise. When the noise had a uniform spectrum the appropriate quantity for picking out the harmonic terms was the periodogram. But when the spectrum of the noise was non-uniform this could no longer be used, and he proposed a method of analysis based on the 
tail of the auto-correlation function; he also showed how a significance test could be constructed.

Mr. B. Landmark (Norwegian Defence Research Establishment) dealt with the provision of a stringent test for the Gaussian character of a given noise signal. An amplitude test was usually insufficient, and he suggested using the simultaneous variations in amplitude and phase. This had been applied experimentally to the scattering from ionospheric clouds ${ }^{1}$, and the results were in good agreement with those to be expected for Gaussian noise.

Dr. L. Mandel (Imperial College of Science and Technology, London) gave an interesting example of a problem in which the approach of the quantum theory produced a substantial simplification. The distribution of the integral, $E_{T}$, over a time interval $T$ of the square of random noise was quite complicated, and some of its properties had been deduced by Rice ${ }^{2}$. If we interpreted the noise as arising from an electromagnetic wave, $E_{T}$ was proportional to the energy contained in a length $c T$ of the wave train. Bose-Einstein statistics could be applied to the photons in this region, and the resulting probability distribution could be determined more readily.

The statistics of radioactive decay was the subject of a communication by Mr. A. C. Hughes. He was concerned with testing fluctuation theory for short. lived substances, that is, those with half-lives short compared with the time of observation. Experiments had been performed with an isotope of rhodium (half-life 44 sec.), and two isotopes of silver (halflives 24 sec. and $2 \cdot 3 \mathrm{~min}$.); the agreement with theory was good.

Dr. M. N. Moore (Birkbeck College) spoke on the "Stochastic Kinetics of Nuclear Reactors". It could be shown that the square of the modulus of the reactor transfer function was proportional to the Fourier transform of the auto-correlation function for power noise in the reactor. Since the power noise represented the response to the minimum power input signal, measurements of transfer functions based upon reactor noise were of all possible measurements least subject to non-linear distortion. By performing the experiment at various power-levels and temperatures, it was possible to measure both power and temperature coefficients ${ }^{3}$.

Some examples were given of new problems in probability which had been suggested by physical phenomena. Prof. C. Domb (King's College, London) said that if one wished to understand what was happening in a regular solution, one must study its fluctuation properties, or the distribution of clusters of different sizes and shapes as a function of temperature. Even for a purely random mixture this was very difficult, although one could readily establish a difference in behaviour in one, two or three dimensions. Thus for a 50:50 mixture if one considered clusters of up to 5 atoms, 88 per cent of the total number were accounted for in one dimension, 17 per eent in two dimensions and only 2 per cent in three dimensions. A critical probability entered in these problems in the same manner as those studied by Hammersley4.

Dr. M. E. Fisher (King's College, London) discussed the shapes and sizes of polymer and polyelectrolyte molecules which seriously affected properties like viscosity. We should be greatly assisted in this field by a knowledge of the properties of non-intersecting random walks on lattices. These walks were nonMarkovian and their behaviour probably differed essentially from Markovian walks. The only property which had been rigorously established ${ }^{5}$ was that $C_{n}$, the total number of walks of $n$ steps, was asymptotically of order $\mu^{n}$. Some conjectures on the value of $\mu$ for a quadratic lattice had been rigorously disproved $^{6}$. The subject suffered seriously from a lack of theorems of the 'central limit' kind. Dr. M. F. Sykes (King's College, London) dealt with methods for the practical determination of parameters in self-avoiding walks. Monte-Carlo methods had been used extensively by Wall and his collaborators? Sykes and his colleagues had preferred to determine the properties exactly for finite values of $n$, rather than to attempt asymptotic extrapolation. When $n$ was larger than about 10 , irregular variations were small, and one could put forward the results with confidence. He estimated that for a quadratic lattice $\mathrm{c}_{n} \sim n^{1 / 3} \mu^{n}$, where $\mu \simeq 2 \cdot 640$ (with a probable error $<\frac{1}{4}$ per cent). Also there was strong evidence that if $p_{n}$ is the number of simple closed polygon walks of $n$ steps, then $p_{n}{ }^{1 / n} \rightarrow \mu$.

Mr. J. M. Hammersley (Atomic Energy Research Establishment, Harwell) spoke on "Percolation Processes"4. These differed from diffusion processes in that the random mechanism was in the medium instead of the fluid. Practical examples of percolation processes were molecules penetrating a porous solid, or disease infecting a community. The processes could be studied in crystals or mazes, and the mathematics was more difficult than that of diffusion processes. It was possible to show rigorously that critical probabilities existed for crystals below which, for example, a fluid starting in one part of the medium would not spread to infinity. Upper and lower limits had been established theoretically for these probabilities, and Monte-Carlo methods had been successfully used to estimate them.

It was not surprising to find several papers devoted to the random walk problem and Brownian movement. Dr. P. H. Roberts (King's College, Newcastle) spoke on the "Random Walk on a Sphere". He was concerned with the geological problem of the path of the Pole as indicated by rocks. Other work in this field had assumed a lattice model, and a planar distribution $^{8 a}$. With the mathematical help of H. D. Ursell, he had used the correct distribution for a sphere, which, incidentally, differed appreciably from the distribution given by R. A. Fisher ${ }^{8 b}$.

The effect of persistence on a random walk was discussed by Mr. A. J. Allnut. In problems such as multiple scattering in foils the assumption that all directions of scatter were equally probable after collision was invalid; it was necessary to take into account persistence in the initial direction, and formulæ for the mean and mean square deviation could readily be derived. Mr. J. C. Barton (Northern Polytechnic, London) described an experimental method of simulating a one-dimensional random walk. This was a problem in which an analogue computer could be of value and could provide information on first passage times for a random walk with persistence ${ }^{9}$. (In the discussion Prof. Bartlett pointed out that theoretical formulæ for first passage times were available for all Markoff processes.)

Dr. A. R. Stokes (King's College, London) in his paper on "Light Scattering by Semi-stiff Chain Molecules" referred to a different application of an analogous problem. The distribution of the end-toend distance of a flexible chain was Gaussian; stiffness in the chain restricted the freedom of the angle between successive links, and modified this distribution, thus influencing the light-scattering 
properties. The modified distribution had been calculated by Daniels ${ }^{10}$, but he had found a simpler approach by using the Fourier transform of the end-to-end distance. The results could be expressed in a form suitable for practical calculation.

The Brownian movement of non-linear systems was discussed by Dr. D. K. C. MacDonald (National Research Council, Ottawa). Many problems in this field still awaited solution. Some results of his own approach $^{11}$ had been substantiated by R. O. Davies ${ }^{12}$, although there was some disagreement with van Kampen ${ }^{13}$, who maintained that the distribution of fluctuations was Gaussian even for non-linear systems. Dr. MacDonald mentioned that he had corresponded with Einstein, who agreed that the statement in his early work on the Gaussian nature of the distribution needed reconsideration.

Dr. A. Suddaby (Sir John Cass College, London) discussed the relation between the microscopic theories of transport processes developed by Kirkwood, and the macroscopic theory of Brownian movement. In the course of his development, Kirkwood introduced a friction constant $\beta$ which was an integral up to time $\tau$ of the correlation of the total force on the particle at different times. The analogous constant in Brownian movement theory was determined by the correlation of the fluctuating force in the Langevin equation. These two values could be shown to agree provided $\beta \tau \ll 1$.

Dr. E. R. Wooding (University of Sheffield) presented a paper on "Recombination in a Plasma as a Stochastic Process". The rate at which ions of opposite charge diffused together was obtained by applying Kramer's method to solve Smoluchowski's equation for diffusion in a field of force. Charge transfer was assumed to occur after the ions approached to within a distance where they could enter a bounded orbit. An ion or atom in the vicinity of an orbit influenced the recombination coefficient. The resulting function was dependent on the degree of ionization, but was similar to that obtained by Thomson ${ }^{14}$ at low pressures, and changed to Jaffe's relationship ${ }^{15}$ at high pressures if the ionization was low.

Dr. G. Wyllie (University of Glasgow) discussed the Brownian motion of spin systems. There were two sources of interest in this problem, its neatness as a model for irreversible processes in quantum mechanics, and the experimental interest in nuclear magnetic resonance experiments. If one focused on individual spins, relaxation times were of the order of milliseconds, whereas for the whole spin system they ranged up to hours. By manipulating electromagnetic fields the spin system could be thrown into conditions far from the Boltzmann distribution. The fluctuating interaction between spins revealed itself in the shape of the magnetic resonance absorption line.

A group of papers was concerned with stochastic problems arising in radio physics. Mr. J. A. Ratcliffe (University of Cambridge) gave an introductory talk, and discussed some problems associated with the Fresnel diffraction patterns formed by an assembly of random irregular diffracting screens. It was well known that, if the correlation function $\rho_{f}(\xi)$ of the complex amplitude $f(x)$ over a onedimensional diffracting screen was defined as $\left\langle f(x) f^{*}(x+\xi)>_{\text {sereen }}\right.$ and if $g(x)$ was the complex amplitude in the diffraction pattern over any plane parallel to the screen, then with certain reservations $\rho_{f}(\xi)$ was equal to $\rho_{g}(\xi)$. If the screen was statistically stationary over $x$ and if the correlation function $r_{f}(\xi)$ was defined as $r_{f}(\xi)=$ $<f\left(x_{1}\right) f^{*}\left(x_{1}+\xi\right)>$ evaluated at two fixed points $x_{1}$ and $\left(x_{1}+\xi\right)$, then it was also true that $r_{g}(\xi)=$ $r_{f}(\xi)=\rho_{f}(\xi)$. If, however, the screen was not statistically stationary the last relation was not necessarily true.

Mr. Ratcliffe considered particularly the nonstationary case when the screen $f(x)$ could be described as an assembly of infinitely long random screens placed, in succession, in front of an aperture of finite width. He suggested that this simple example represented approximately the problems of the diffraction of $(a)$ radio waves radiated from a radio star with a sharp boundary, $(b)$ light waves radiated from a source of light placed behind a slit, or (c) radio waves reflected from an irregular meteor trail of limited length. He stated that, if the limiting aperture subtended less than the first Fresnel zone at the observing plane, then $r_{g}(\xi)$ was determined, not by the fine structure in the screen, but by the aperture bounding it. If, however, the aperture subtended a large number of Fresnel zones then $r_{g}(\xi)=r_{f}(\xi)$ and was determined by the fine structure in the screen.

Mr. S. A. Bowhill (Pennsylvania State University) discussed the scattering of electromagnetic waves from a continuous medium containing three-dimensional random inhomogeneities of refractive index. He had derived the form of the emerging angular power spectrum when the scales of the inhomogeneities were different in the three space directions. Contrary to previous results ${ }^{16}$, he had found that the medium could not be analysed as a series of superposed thin phase screens, spaced in the propagation direction, and with independent phase profiles.

Mr. M. L. V. Pitteway (University of Cambridge) was concerned with reflexion from an irregular medium. Before proceeding to a three-dimensional solution for irregularities he thought one should obtain a solution for a horizontally stratified ionosphere ${ }^{17}$ and treat the irregularities as a small perturbation. The power spectrum of the scattered wave could then be expressed as an integral in terms of the stratified solution. Dr. B. H. Briggs (University of Cambridge) dealt with the experimental problem of specifying the pattern on the ground (including time changes and movements) formed by reflexion from, or transmission through, an irregular ionosphere. He defined parameters which could be used to specify this pattern, and which could be deduced from observations at a few points on the ground ${ }^{18}$. As an example, he considered the application to radio star scintillations. Mr. R. P. Mercier (University of Cambridge) discussed theoretical aspects of radio-wave fading. A scalar wave with random variations of amplitude and phase across the wave front was taken as a simple model. It was assumed that the in-phase and quadrature components of the fluctuating part of the field were normally distributed, and a parameter was introduced to specify the intrinsic correlation of the fading. Various properties of this parameter could be derived, and used to interpret fading from the ionosphere.

"Coherence Properties of Partially Polarized Light" was the subject of the paper by Dr. E. Wolf (University of Manchester). Observing that the usual definition of Stokes parameters of a quasi-mono. chromatic plane electric wave was not unique, an experiment was analysed which led to a unique 
coherency matrix and to a unique set of Stokes parameters. The degree of polarization of such a wave was also equal to the maximum value of the degree of coherence which existed between the components of electric vibrations in orthogonal directions in the wave front. This suggested a new method of measurement of the degree of polarization, based on interference experiments.

Prof. E. G. Richardson (King's College, Newcastle) referred to experiments on the propagation of sound waves in a fluid having random variations in either density or momentum, whereby the amplitude and relative phase of the signal picked up after transmission through the medium fluctuated in time. The former type occurred near the critical point of a fluid or of a mixture of liquids, the latter in the atmosphere or in the wake of an obstacle or, again, in a boundary layer. Analyses of such measurements were presented. In the case of the liquid mixture a correlation was sought between the pattern of the scattered radiation and the mean size of the clusters which formed at the critical point. As an example of the second type, frequency spectra of the modulations of the sound signal transmitted athwart the wake of a cylinder involved the discerning of peaks in the spectrum against the background of fluctuation 'noise' in the general flow.

Dr. M. S. Longuet-Higgins (National Institute of Oceanography) discussed "Sea-Waves as a Stochastic Process". He showed a typical record of pressure at a fixed point on the sea bed which agreed elosely with a Gaussian distribution. Non-Gaussian features usually appeared when the waves were steep and near the point of breaking, or in shallow water. To describe the sea surface a random process two spatial dimensions and one of time were needed; the practical problem for wave forecasting was to relate this to winds and other relevant factors. Dr. Longuet-Higgins also listed a number of properties of a Gaussian surface which might be of use in determining the spectrum; these included wave slopes ${ }^{19}$, 'specular points' and 'twinkles'20.

The final paper was given by Prof. E. W. Montroll (University of Maryland) on a stochastic treatment of traffic flow. Experimental data indicated that the acceleration of a car in a line of traffic at time $t$ was proportional to the velocity difference between itself and its neighbour at time $(t-\Delta)$, where $\Delta \sim 1.5$ sec. and the proportionality constant ${ }^{21}$ was 0.37 sec. $^{-1}$. Theoretical investigation showed that the motion became unstable when the product of lag time and proportionality constant exceeded $1 / 2$. Thus the experimental data showed that driving was usually on the verge of instability. 'Acceleration noise' was put forward as a parameter which would characterize the driver - car - road complex under various con. ditions ${ }^{22}$. Reasonable agreement was obtained with traffic flow measurements ${ }^{23}$.

The conference was organized at the suggestion of Dr. Furth, who is to be highly commended on his initiative. The one hundred participants would undoubtedly wish to express their thanks to him, to Birkbeck College, and to the Physical Society for the excellent arrangements.

C. Dомв

${ }^{1}$ Hagfors, T., and Landmark, B., Proc. Inst. Elec. Eng., B, 105, 555 (1958).

'Rice, S. O., Bell Syst. Tech. J., 23, 282 (1944).

${ }^{3}$ Moore, M. N., Nuclear Science and Engineering, 3, 387 (1958).

4 Broadbent, S. R., and Hammersley, J. M., Proc. Camb. Phil. Soc. 53, 629 (1957). Hammersley, J. M., ibid., 53, 642 (1957); Ann. Math. Statist., 28, 790 (1057)

${ }^{5}$ Hammersley, J. M., and Morton, K. W., J. Roy. Stat. Soc., B, 16. 23 (1954).

${ }^{6}$ Fisher, M. E., Farad. Soc. Disc., 25, 200 (1958).

'Wall, F. T., Hiller, L. A., and Atchison, W. F., J. Chem. Phys., 26 1742 (1957). Wall, F. T., Rubin, R. J., and Isaacson, L. M. J. Chem. Phys., 27, 186 (1957).

${ }^{8}$ Fisher, M. E., and Sykes, M. F., Phys. Rev., 114, 45 (1959). ${ }^{8} a$ Green, R., Nature, 182, 382 (1958). "'s Fisher, R. A., Proc. Roy. Soc., A, 217. 295 (1953).

- Barton, J. C., Campbell, D. A., and Read, R. C., Proc. Phys. Soc., A, 70,605 (1957).

${ }^{10}$ Daniels, H. E., Proc. Roy. Soc. Edin., 63, 290 (1952).

$"$ MacDonald, D. K. C., Phys. Rev., 108, 541 (1957).

${ }_{12}$ Davies, R. O., Physica, 24, 1055 (1958).

${ }^{13}$ Van Kampen, N. G., Phys. Rev., 110, 320 (1.958).

${ }^{14}$ Thomson, J. J., Phil. Mag., 47, 337 (1924).

${ }^{15}$ Jaffe, G., Phys. Rev., 58, 968 (1940).

${ }^{16}$ Fejer, J. A., Proc. Roy. Soc., A, 220, 445 (1953). Feinstein, J. Trans. Inst. Rad. Eng., APे-2, 23, 63 (1954). Bramley, E. N.' Proc. Roy. Soc., A, 225, 515 (1954).

${ }^{17}$ Pitteway, M. L. V., Proc. Roy. Soc., A, 246, 556 (1958).

${ }_{18}$ Briggs, B. H., Phillips, G. J., and Shinn, D. H., Proc. Phys. Soc. B, 68, 106 (1950). Phillips, G. J., and Spencer, M., ibid., 68, 481 (1955).

19 Cox, C., and Munk, W., J. Opt. Soc. Amer., 44, 838 (1954).

${ }^{20}$ Longuet-Higgins, M. S., Phil. Trans. Roy. Soc., A, 249, 321 (1957); 250,157 (1957) ; Proc. Camb. Phil. Soc., 55, 91 (1959).

${ }^{21}$ Chandler, R. E., Herman, R., and Montroll, E. W., Operations Res., 6, 165 (1958)

${ }^{2}$ Herman, R., Montroll, E. W., Potts, R. B., and Rothery, R. W. Operations Res., 7,86 (1959).

${ }^{23}$ Greenberg, H., Operations Res., 7, 79 (1959).

\section{BIOMECHANICS}

$\mathrm{O}^{\mathrm{N}}$ April 17 the Institution of Mechanical Engineers held a symposium on "Biomechanics" with the purpose of bringing medical men and engineers into closer contact.

Biomechanics, in its broadest sense, may be defined as the branch of science which applies the principles of mechanics and the techniques of engineering to the human body in the process of its repair, and in the field of man-machine relationship, where man is the essential link in operating these machines.

The symposium was opened by the President of the Institution and the papers and discussion were presented under the chairmanship of Prof. S. J. Davies. In the opening paper on the importance of biomechanics as a service to man, illustrated by a discussion of problems in metallic osteosynthesis, J. M. Zarek, from King's College, London, gave a brief account of the nature of biomechanics as a new field of endeavour in which some of the engineering knowledge may be of direct use to the medical man. The man - machine relationship was only mentioned, as this aspect of biomechanics appears to be already well appreciated by the engineers and was not dealt with at the symposium in detail. After discussing the scope of biomechanics, the general history and current British activities in this field were reviewed. Problems of bone repair were considered at length and the work in the Civil Engineering Department at King's College, London, on the stress/bone formation relationship and the behaviour of metals in the human body were diseussed. 